\title{
SOME RESULTS OF SECOND ORDER DIFFERENTIAL SUBORDINATION INVOLVING GENERALIZED LINEAR OPERATOR
}

\author{
A.R.S. Juma, R.A. Hameed, M.I. Hameed
}

ABSTRACT. In this paper, we derive some results of second order differential subordination involving linear operator, we estimate some of the interesiting property in would provide extensions of those given in earlier works.

2010 Mathematics Subject Classification: 30C45, 30A20, 34A40.

Keywords: Univalent function, Differential subordination, Differential superordination, Convex function, Best dominant, Generalized operator and Dziok-Srivastava operator.

\section{INTRODUCTION}

Let $H(U)$ denote the class of holomorphic functions in the open unit disc $U=\{z \in$ $C:|z|<1\}$ in $\mathrm{C}$ be complex plane. Let $\mathrm{A}$ be the class of analytic functions normalized by $\varphi(0)=0$ and $\varphi(0)=1$ in $U$ of the following form

$$
\varphi(z)=z+\sum_{n=2}^{\infty} a_{n} z^{n},
$$

with $A_{1}=A$, where $a \in C$ and $n \in N=\{1,2, \ldots\}$, we let

$$
H[a, n]=\left\{\varphi \in H(U): \varphi(z)=a+a_{n} z^{n}+a_{n+1} z^{n+1}+\ldots\right\},
$$

be the subclass of $H(U)$ with $H_{0} \equiv H[0,1]$ and $H \equiv H[1,1]$.

Next, let $\varphi(z)$ and $F(z)$ be members of $H(U)$. Then the function $\varphi(z)$ is said to be subordinate to a function $F(z)$ or $F(z)$ is said to be superordinate to $\varphi(z)$, written as

$$
\varphi(z) \prec F(z) \text { or } \varphi \prec F,
$$


A.R.S. Juma, R.A. Hameed, M.I. Hameed - Some Results of Second Order ...

if and only if if there exists a Schwarz function $w(z)$ holomorphic in $U$, with $w(0)=0$ and $|w(z)|<1,(z \in U)$, such that $\varphi(z)=F(w(z))$.

Furthermore, if the function $\mathrm{F}$ is univalent in $\mathrm{U}$, then we have the following equivalence $[3],[7],[9]$.

$$
\varphi(z) \prec F(z) \Longleftrightarrow \varphi(0)=F(0) \text { and } \varphi(U) \subset F(U) .
$$

Let $S$ denote the subclass of $A$ consisting of functions univalent in $U$. If a function $\varphi \in A$ maps $U$ onto a convex domain and $\varphi$ is univalent, then $\varphi$ is called a convex function. Let

$$
K=\left\{\varphi \in A: \operatorname{Re}\left(1+\frac{z \varphi^{\prime}(z)}{\dot{\varphi}(z)}>0\right), z \in U\right\},
$$

denote the class of all convex functions defined in $U$ and normalized by $\varphi=0$ and $\varphi^{\prime}(0)=1$.

Let $\phi: C^{3} \times U \rightarrow C$ be holomorphic in a domain $D$ and $g$ be univalent in $U$. If $h$ is holomorphic in $U$ and $h(0)=a$ with satisfies the second-order differential subordination

$$
\phi\left(h(z), z h^{\prime}(z), z h^{\prime}(z) ; z\right) \prec g(z),
$$

then $h$ is said to be solution of the differential subordination. The univalent function $p$ is said to be dominant of the solution of the differential subordination or more simply dominant, if $h \prec p$ for all $h$ satisfying (2). A dominant $p^{\sim}$ satisfying $p^{\sim} \prec p$ for all dominants (2) is said to be the best dominant of (2).

Now, we defined the new generalized studied by

Definition 1.1. [1] For $\varphi \in A$ the generalized derivative operator $I_{\lambda, \eta}^{m, s}: A \rightarrow A$ is defined by.

$$
I_{\lambda, \eta}^{m, s} \varphi(z)=z+\sum_{n=2}^{\infty} \frac{(1+\lambda(n-1))^{m}}{(1+\eta(n-1))^{m-1}} c(s, n) a_{n} z^{n},
$$

where $s, m \in N_{0}=\{0,1, \ldots\}, \eta \geq \lambda \geq 0$ and $c(s, n)=\frac{(s+1)_{n-1}}{(1)_{n-1}}$.

It can be easily seen that

$$
I_{\lambda, 0}^{0,0} \varphi(z)=I_{0, \eta}^{1,0} \varphi(z)=\varphi(z)
$$

and

$$
I_{\lambda, 0}^{1,0} \varphi(z)=I_{0, \eta}^{1,1} \varphi(z)=z \dot{\varphi}(z)
$$


A.R.S. Juma, R.A. Hameed, M.I. Hameed - Some Results of Second Order ...

Also,

$$
I_{\lambda, 0}^{b-1,0} \varphi(z)=I_{0, \eta}^{1, b-1} \varphi(z) \text { where } b=1,2,3, \ldots
$$

We can verify that

$$
(1+s) I_{\lambda, \eta}^{m, s+1} \varphi(z)=z\left(I_{\lambda, \eta}^{m, s} \varphi(z)\right)^{\prime}+s\left(I_{\lambda, \eta}^{m, s} \varphi(z)\right) .
$$

Definition 1.2. [13] For $\varphi \in A$, the Dziok-Srivastava operator $H_{m}^{i}: A \rightarrow A$ is defined by

$H_{m}^{i}\left(\alpha_{1}, \alpha_{2}, \ldots, \alpha_{i} ; \beta_{1}, \beta_{2} \ldots, \beta_{m}\right) \varphi(z)=z+\sum_{n=2}^{\infty} \frac{\left(\alpha_{1}\right)_{n-1}\left(\alpha_{2}\right)_{n-1} \ldots\left(\alpha_{i}\right)_{n-1}}{\left(\beta_{1}\right)_{n-1}\left(\beta_{2}\right)_{n-1} \ldots\left(\beta_{m}\right)_{n-1}} \frac{1}{(n-1) !} a_{n} z^{n}$

where $\alpha_{i} \in C, i=1,2, \ldots, i, \beta_{j} \in C \backslash\{0,-1,-2, \ldots\}, j=1,2, . ., m$.

For complex numbers $a, b$ and $c$ other than $0,-1,-2, \ldots$, the Gauss hypergeometric function ${ }_{2} F_{1}(a, b, c ; z)$ is defined by

$$
{ }_{2} F_{1}(a, b, c ; z)=\sum_{n=0}^{\infty} \frac{(a)_{n}(b)_{n}}{(c)_{n}} \frac{z^{n}}{n !}=\frac{\Gamma(c)}{\Gamma(a) \cdot \Gamma(b)} \sum_{n=0}^{\infty} \frac{\Gamma(a+n) \Gamma(b+n)}{\Gamma(c+n)} \frac{z^{n}}{n !}
$$

where $(x)_{n}$ is the Pochhammer symbol defined, in terms of the Gamma function $\Gamma$, by

$$
(x)_{n}=\frac{\Gamma(x+n)}{\Gamma(x)}=\left\{\begin{array}{l}
x(x+1)(x+2) \ldots(x+n-1) \text { for } n \in \text { Nand } x \in C, \\
1, \text { if } n=0 \text { and } x \in C \backslash\{0\} .
\end{array}\right.
$$

In order to make the notation simple, we write

$$
H_{m}^{i}\left[\alpha_{1}\right] \varphi(z)=H_{m}^{i}\left(\alpha_{1}, \alpha_{2}, \ldots, \alpha_{i} ; \beta_{1}, \beta_{2}, \beta_{m}\right) \varphi(z) .
$$

We can verify that

$$
\alpha_{1} H_{m}^{i}\left[\alpha_{1}+1\right] \varphi(z)=z\left(H_{m}^{i}\left[\alpha_{1}\right] \varphi(z)\right)^{\prime}+\left(\alpha_{1}-1\right) H_{m}^{i}\left[\alpha_{1}\right] \varphi(z) .
$$

Here, we defined the following.

Definition 1.3. For $\varphi \in A$ the operator $I H_{m}^{i}: A \rightarrow A$ is defined by the Hadamard product of the generalized operator $I_{\lambda, \eta}^{m, s}$ and the Dziok-Srivastava operator $H_{m}^{i}$.

$$
I H_{m}^{i} \varphi(z)=\left(I_{\lambda, \eta}^{m, s} * H_{m}^{i}\right) \varphi(z)(z \in U),
$$

and

$$
I H_{m}^{i} \varphi(z)=z+\sum_{n=2}^{\infty} \frac{(1+\lambda(n-1))^{m}}{(1+\eta(n-1))^{m-1}} c(s, n) \frac{\left(\alpha_{1}\right)_{n-1}\left(\alpha_{2}\right)_{n-1} \ldots\left(\alpha_{i}\right)_{n-1}}{\left(\beta_{1}\right)_{n-1}\left(\beta_{2}\right)_{n-1} \ldots\left(\beta_{m}\right)_{n-1}} \frac{1}{(n-1) !} a_{n}^{2} z^{n} .
$$


A.R.S. Juma, R.A. Hameed, M.I. Hameed - Some Results of Second Order ...

Not that, the following are the special cases of operator $I H_{m}^{i}$.

1. When $I_{0,0}^{m, 0}=1$, inclued the Dziok-Srivastava operator $H_{m}^{i}[5],[13]$.

2. When $i=2, m=1, H_{1}^{2}$ which is introduced by the Gauss hypergeometric function [2].

3. When $H_{1}^{1}=1$, inclued the generalized derivative operator $I_{\lambda, \eta}^{m, s}[1]$.

4. When $s=0, \lambda=1, \eta=0, I_{\lambda, \eta}^{m, s}$ reduces to $I_{1,0}^{m, 0}$ which is introduced by Salagean derivative operator $[8]$.

5. When $s=0, \eta=0, I_{\lambda, \eta}^{m, s}$ reduces to $I_{\lambda, 0}^{m, 0}$ which is introduced by generalized Salagean derivative operator introduced by Al-oboudi [11].

6. When $\eta=0, I_{\lambda, \eta}^{m, s}$ reduces to $I_{\lambda, 0}^{m, s}$ which is introduced by generalized Al-Shaqsi and Darus derivative operator [4].

7. When $\lambda=0, \eta=0, I_{\lambda, \eta}^{m, s}$ reduces to $I_{0,0}^{m, s}$ which is introduced by SrivastavaAttiya derivative operator [15].

8. When $m=1$ or $m=0, \lambda=0$ or $\lambda=\lambda, \eta=\eta$ or $\eta=0, I_{\lambda, \eta}^{m, s}$ reduces to $I_{0, \eta}^{1, s} ? I_{\lambda, 0}^{0, s}$ which is introduced by Ruscheweyh derivative operator [12].

9. When $m=0$ or $m=1, I_{\lambda, \eta}^{m, s}$ reduces to $I_{\lambda, \eta}^{0, s} ? I_{\lambda, \eta}^{1, s}$ which is introduced by generalized Ruscheweyh derivative operator [14].

In order to prove the results, we need the following lemmas.

Lemma 1.1. [6] If $q$ is holomorphic, univalent and convex function in $U$, let $q(0)=a$ and $\gamma \in C^{*}=C \backslash\{0\}$ be a complex number such that $\operatorname{Re}\{\gamma\} \geq 0$. If $p \in H[a, n]$ and

$$
p(z)+\frac{z p^{\prime}(z)}{\gamma} \prec q(z),
$$

then

$$
p(z) \prec h(z) \prec q(z),
$$

where

$$
h(z)=\frac{\gamma}{n z^{\frac{\gamma}{n}}} \int_{0}^{z} q(t) t^{\frac{\gamma}{n}-1} d t
$$

The function $h$ is convex and it is the best $(a, n)$-dominant of the subordination (9). 
A.R.S. Juma, R.A. Hameed, M.I. Hameed - Some Results of Second Order ...

Lemma 1.2. [10] If $r$ is a convex function in $U$ with

$$
h(z)=r(z)+n \beta z r^{\prime}(z) \quad(z \in U),
$$

where $\beta>0$ and $n \in N$. If

$$
p(z)=r(0)+p_{n} z^{n}+p_{n+1} z^{n+1}+, \quad(z \in U),
$$

is holomorphic in $U$ and

$$
p(z)+\beta z p^{\prime}(z) \prec h(z),
$$

then

$$
p(z) \prec r(z),
$$

and this result is sharp.

\section{Main Results}

Theorem 2.1. If $h$ be a convex function with $h(0)=1$ with $q$ be the following function

$$
q(z)=h(z)+\frac{z}{\tau} h^{\prime}(z), \tau, i \geq 0, m \in N .
$$

If $\varphi \in A$ and the following differential subordination

$$
\left(\frac{I H_{m}^{i} \varphi(z)}{z}\right)^{\tau-1}\left(I H_{m}^{i} \varphi(z)\right)^{\prime} \prec q(z)
$$

holds true, then

$$
\left(\frac{I H_{m}^{i} \varphi(z)}{z}\right)^{\tau} \prec h(z),
$$

Proof. If $\varphi \in A$, given by (1) therefore, we get

$I H_{m}^{i} \varphi(z)=z+\sum_{n=2}^{\infty} \frac{(1+\lambda(n-1))^{m}}{(1+\eta(n-1))^{m-1}} c(s, n) \frac{\left(\alpha_{1}\right)_{n-1}\left(\alpha_{2}\right)_{n-1} \ldots\left(\alpha_{i}\right)_{n-1}}{\left(\beta_{1}\right)_{n-1}\left(\beta_{2}\right)_{n-1} \ldots\left(\beta_{m}\right)_{n-1}} \frac{1}{(n-1) !} a_{n}^{2} z^{n}, \quad(z \in U)$

let us consider

$$
\begin{array}{r}
p(z)=\left(\frac{I H_{m}^{i} \varphi(z)}{z}\right)^{\tau}=\left(\frac{z+\sum_{n=2}^{\infty} \frac{(1+\lambda(n-1))^{m}}{(1+\eta(n-1))^{m-1}} c(s, n) \frac{\left(\alpha_{1}\right)_{n-1}\left(\alpha_{2}\right)_{n-1 \ldots} \ldots\left(\alpha_{i}\right)_{n-1}}{\left(\beta_{1}\right)_{n-1}\left(\beta_{2}\right)_{n-1} \ldots\left(\beta_{m}\right)_{n-1}} \frac{1}{(n-1) !} a_{n}^{2} z^{n}}{z}\right)^{\tau} \\
=\left(1+\sum_{n=2}^{\infty} \frac{(1+\lambda(n-1))^{m}}{(1+\eta(n-1))^{m-1}} c(s, n) \frac{\left(\alpha_{1}\right)_{n-1}\left(\alpha_{2}\right)_{n-1} \ldots\left(\alpha_{i}\right)_{n-1}}{\left(\beta_{1}\right)_{n-1}\left(\beta_{2}\right)_{n-1} \ldots\left(\beta_{m}\right)_{n-1}} \frac{1}{(n-1) !} a_{n}^{2} z^{n-1}\right)^{\tau}
\end{array}
$$


A.R.S. Juma, R.A. Hameed, M.I. Hameed - Some Results of Second Order ...

$$
p(z)=1+p_{\tau} z^{\tau}+p_{\tau+1} z^{\tau+1}+\ldots .
$$

By take the derivative of both sides, we get

$$
\left(\frac{I H_{m}^{i} \varphi(z)}{z}\right)^{\tau-1}\left(I H_{m}^{i} \varphi(z)\right)^{\prime}=p(z)+\frac{z p^{\prime}(z)}{\tau} .
$$

Thus, by aid of the relation (10) becomes

$$
p(z)+\frac{z p^{\prime}(z)}{\tau} \prec q(z)=h(z)+\frac{z h^{\prime}(z)}{\tau},(z \in U)
$$

therefore, with Lemma 1.2 we obtain

$$
p(z) \prec h(z),
$$

that is,

$$
\left(\frac{I H_{m}^{i} \varphi(z)}{z}\right)^{\tau} \prec h(z) .
$$

Theorem 2.2. If $q$ be a holomorphic function and satisfies in the following inequality

$$
\operatorname{Re}\left\{1+\frac{z q^{\prime}(z)}{q^{\prime}(z)}\right\}>-1 / 2, \text { where } q(0)=1, \tau, i \geq 0, m \in N,
$$

and $\varphi \in A$ satisfies in the following differential subordination

$$
\left(\frac{I H_{m}^{i} \varphi(z)}{z}\right)^{\tau-1}\left(I H_{m}^{i} \varphi(z)\right)^{\prime} \prec q(z) .
$$

Then

$$
\left(\frac{I H_{m}^{i} \varphi(z)}{z}\right)^{\tau} \prec g(z)
$$

where

$$
g(z)=\frac{\tau}{z^{\tau}} \int_{0}^{z} q(t) t^{\tau-1} d t
$$


A.R.S. Juma, R.A. Hameed, M.I. Hameed - Some Results of Second Order ...

Proof. Setting

$$
\begin{aligned}
p(z)=\left(\frac{I H_{m}^{i} \varphi(z)}{z}\right)^{\tau}=\left(\frac{z+\sum_{n=2}^{\infty} \frac{(1+\lambda(n-1))^{m}}{(1+\eta(n-1))^{m-1}} c(s, n) \frac{\left(\alpha_{1}\right)_{n-1}\left(\alpha_{2}\right)_{n-1} \ldots\left(\alpha_{i}\right)_{n-1}}{\left(\beta_{1}\right)_{n-1}\left(\beta_{2}\right)_{n-1} \ldots\left(\beta_{m}\right)_{n-1}} \frac{1}{(n-1) !} a_{n}^{2} z^{n}}{z}\right)^{\tau} \\
=\left(1+\sum_{n=2}^{\infty} \frac{(1+\lambda(n-1))^{m}}{(1+\eta(n-1))^{m-1}} c(s, n) \frac{\left(\alpha_{1}\right)_{n-1}\left(\alpha_{2}\right)_{n-1} \ldots\left(\alpha_{i}\right)_{n-1}}{\left(\beta_{1}\right)_{n-1}\left(\beta_{2}\right)_{n-1} \ldots\left(\beta_{m}\right)_{n-1}} \frac{1}{(n-1) !} a_{n}^{2} z^{n-1}\right)^{\tau}, \\
p(z)=1+p_{\tau} z^{\tau}+p_{\tau+1} z^{\tau+1}+\ldots=1+\sum_{n=\tau+1}^{\infty} p_{n} z^{n-1}, p \in H[1, \tau] .
\end{aligned}
$$

By take the derivative of both sides, we get

$$
\left(\frac{I H_{m}^{i} \varphi(z)}{z}\right)^{\tau-1}\left(I H_{m}^{i} \varphi(z)\right)^{\prime}=p(z)+\frac{z p^{\prime}(z)}{\tau},
$$

thus, by aid of the relation the relation (10) becomes

$$
p(z)+\frac{z p^{\prime}(z)}{\tau} \prec q(z),
$$

therefore, with Lemma 1.2 we obtain

$$
p(z) \prec g(z),
$$

that is,

$$
\left(\frac{I H_{m}^{i} \varphi(z)}{z}\right)^{\tau} \prec g(z),
$$

where

$$
g(z)=\frac{\tau}{z^{\tau}} \int_{0}^{z} q(t) t^{\tau-1} d t .
$$

The function $g(z)$ is convex and it is the best dominant.

Corollary 2.3. If the function $q$ defined by

$$
q(z)=\frac{1+(2 \alpha-1) z}{1+z},(0 \leq \alpha<1)
$$

be a convex function in $U$, where $\tau, i \geq 0, m \in N$ and $\varphi \in A$ be such that

$$
\left(\frac{I H_{m}^{i} \varphi(z)}{z}\right)^{\tau-1}\left(I H_{m}^{i} \varphi(z)\right)^{\prime} \prec q(z),
$$


A.R.S. Juma, R.A. Hameed, M.I. Hameed - Some Results of Second Order ...

holds, then

$$
\left(\frac{I H_{m}^{i} \varphi(z)}{z}\right)^{\tau} \prec g(z)
$$

$g(z)$ is given by $g(z)=(2 \alpha-1)+\frac{2(1-\alpha) \tau}{z^{\tau}} \int_{0}^{z} \frac{t^{\tau-1}}{1+t} d t$. The function $g(z)$ is convex and it is the best dominant.

Proof. Putting

$$
p(z)=\left(\frac{I H_{m}^{i} \varphi(z)}{z}\right)^{\tau},
$$

thus, by aid of the relation (10) becomes

$$
p(z)+\frac{z p^{\prime}(z)}{\tau} \prec q(z)=\frac{1+(2 \alpha-1) z}{1+z},(0 \leq \alpha<1)
$$

therefore, with Lemma 1.1 for $\gamma=\tau$, we obtain

$$
\begin{array}{r}
\left(\frac{I H_{m}^{i} \varphi(z)}{z}\right)^{\tau} \prec g(z)=\frac{\tau}{z^{\tau}} \int_{0}^{z} q(t) t^{\tau-1} d t=\frac{\tau}{z^{\tau}} \int_{0}^{z} t^{\tau-1} \frac{1+(2 \alpha-1) t}{1+t} d t \\
=\frac{\tau}{z^{\tau}} \int_{0}^{z}\left[(2 \alpha-1) t^{\tau-1}+2(1-\alpha) \frac{t^{\tau-1}}{1+t}\right] d t=(2 \alpha-1)+\frac{2(1-\alpha) \tau}{z^{\tau}} \int_{0}^{z} \frac{t^{\tau-1}}{1+t} d t .
\end{array}
$$

Theorem 2.4. If $h$ is a convex function with $h(0)=1$ such that $q$ the following function

$$
q(z)=h(z)+\frac{z}{\tau} h^{\prime}(z), \quad(z \in U)
$$

where $\tau, i \geq 0, m \in N$.If $\varphi \in A$ and satisfies the following differential subordination

$$
z \frac{\tau+1}{\tau} \frac{I H_{m}^{i} \varphi(z)}{\left(I H_{m}^{i}\left[\alpha_{1}+1\right] \varphi(z)\right)^{2}}+\frac{z^{2}}{\tau} \frac{I H_{m}^{i} \varphi(z)}{\left(I H_{m}^{i}\left[\alpha_{1}+1\right] \varphi(z)\right)^{2}}\left[\frac{\left(I H_{m}^{i} \varphi(z)\right\}}{I H_{m}^{i} \varphi(z)}-2 \frac{\left(I H_{m}^{i}\left[\alpha_{1}+1\right] \varphi(z) \hat{f}\right.}{I H_{m}^{i} \varphi(z)}\right] \prec q(z),
$$

then

$$
z \frac{I H_{m}^{i} \varphi(z)}{\left(I H_{m}^{i}\left[\alpha_{1}+1\right] \varphi(z)\right)^{2}} \prec h(z)
$$

The result is sharp. 
A.R.S. Juma, R.A. Hameed, M.I. Hameed - Some Results of Second Order ...

Proof. If $\varphi \in A$, given by (1) therefore, we get

$I H_{m}^{i} \varphi(z)=z+\sum_{n=2}^{\infty} \frac{(1+\lambda(n-1))^{m}}{(1+\eta(n-1))^{m-1}} c(s, n) \frac{\left(\alpha_{1}\right)_{n-1}\left(\alpha_{2}\right)_{n-1} \ldots\left(\alpha_{i}\right)_{n-1}}{\left(\beta_{1}\right)_{n-1}\left(\beta_{2}\right)_{n-1} \ldots\left(\beta_{m}\right)_{n-1}} \frac{1}{(n-1) !} a_{n}^{2} z^{n}$,

let us consider

$$
p(z)=z \frac{I H_{m}^{i} \varphi(z)}{\left(I H_{m}^{i}\left[\alpha_{1}+1\right] \varphi(z)\right)^{2}}
$$

we have

$$
\begin{gathered}
p(z)+\frac{z}{\tau} p^{\prime}(z)= \\
z \frac{\tau+1}{\tau} \frac{I H_{m}^{i} \varphi(z)}{\left(I H_{m}^{i}\left[\alpha_{1}+1\right] \varphi(z)\right)^{2}}+\frac{z^{2}}{\tau} \frac{I H_{m}^{i} \varphi(z)}{\left(I H_{m}^{i}\left[\alpha_{1}+1\right] \varphi(z)\right)^{2}}\left[\frac{\left(I H_{m}^{i} \varphi(z)\right)^{\prime}}{I H_{m}^{i} \varphi(z)}-2 \frac{\left(I H_{m}^{i}\left[\alpha_{1}+1\right] \varphi(z)\right)^{\prime}}{I H_{m}^{i} \varphi(z)}\right] .
\end{gathered}
$$

Thus, by aid of the relation (11) becomes

$$
p(z)+\frac{z}{\tau} p^{\prime}(z) \prec q(z)=h(z)+\frac{z}{\tau} h^{\prime}(z), \quad(z \in U)
$$

therefore, with Lemma 1.2 we obtain

$$
p(z)=z \frac{I H_{m}^{i} \varphi(z)}{\left(I H_{m}^{i}\left[\alpha_{1}+1\right] \varphi(z)\right)^{2}} \prec h(z) .
$$

Theorem 2.5. For $\varphi \in A$ and $q$ be a holomorphic function and it satisfies the inequality

$$
\operatorname{Re}\left\{1+\frac{z q^{\prime}(z)}{q^{\prime}(z)}\right\}>-1 / 2, \text { with } q(0)=1, \tau, i \geq 0, m \in N,
$$

and it satisfies the following differential subordination

$z \frac{\tau+1}{\tau} \frac{I H_{m}^{i} \varphi(z)}{\left(I H_{m}^{i}\left[\alpha_{1}+1\right] \varphi(z)\right)^{2}}+\frac{z^{2}}{\tau} \frac{I H_{m}^{i} \varphi(z)}{\left(I H_{m}^{i}\left[\alpha_{1}+1\right] \varphi(z)\right)^{2}}\left[\frac{\left(I H_{m}^{i} \varphi(z)\right)^{\prime}}{I H_{m}^{i} \varphi(z)}-2 \frac{\left(I H_{m}^{i}\left[\alpha_{1}+1\right] \varphi(z)\right)^{\prime}}{I H_{m}^{i} \varphi(z)}\right] \prec q(z)$,

then

$$
z \frac{I H_{m}^{i} \varphi(z)}{\left(I H_{m}^{i}\left[\alpha_{1}+1\right] \varphi(z)\right)^{2}} \prec g(z)
$$

where $g(z)$ is given by

$$
g(z)=\frac{\tau}{z^{\tau}} \int_{0}^{z} q(t) t^{\tau-1} d t
$$

The function $g(z)$ is convex and it is the best dominant. 
A.R.S. Juma, R.A. Hameed, M.I. Hameed - Some Results of Second Order ...

Proof. Putting

$$
p(z)=z \frac{I H_{m}^{i} \varphi(z)}{\left(I H_{m}^{i}\left[\alpha_{1}+1\right] \varphi(z)\right)^{2}}, p \in H[1,1] .
$$

By take the derivative of both sides, we get

$$
p(z)+\frac{z}{\tau} p(z)=
$$

$z \frac{\tau+1}{\tau} \frac{I H_{m}^{i} \varphi(z)}{\left(I H_{m}^{i}\left[\alpha_{1}+1\right] \varphi(z)\right)^{2}}+\frac{z^{2}}{\tau} \frac{I H_{m}^{i} \varphi(z)}{\left(I H_{m}^{i}\left[\alpha_{1}+1\right] \varphi(z)\right)^{2}}\left[\frac{\left(I H_{m}^{i} \varphi(z)\right)^{\prime}}{I H_{m}^{i} \varphi(z)}-2 \frac{\left(I H_{m}^{i}\left[\alpha_{1}+1\right] \varphi(z)\right)^{\prime}}{I H_{m}^{i} \varphi(z)}\right]$,

thus, by aid of the relation (11) becomes

$$
p(z)+\frac{z}{\tau} p(z) \prec q(z) .
$$

Therefore, with Lemma 1.1 we obtain

$$
p(z)=z \frac{I H_{m}^{i} \varphi(z)}{\left(I H_{m}^{i}\left[\alpha_{1}+1\right] \varphi(z)\right)^{2}} \prec g(z) .
$$

The function $g(z)$ is convex and it is the best dominant.

Theorem 2.6. If $h$ is a convex function with $h(0)=1$ such that $q$ the following function

$$
q(z)=h(z)+\frac{z}{\tau} h^{\prime}(z), \quad(z \in U)
$$

where $\tau, i \geq 0, m \in N$.If $\varphi \in A$ and satisfies the following differential subordination

$$
z^{2} \frac{\tau+2}{\tau} \frac{\left(I H_{m}^{i} \varphi(z)\right)^{\prime}}{I H_{m}^{i} \varphi(z)}+\frac{z^{3}}{\tau}\left[\frac{\left(I H_{m}^{i} \varphi(z)\right)^{\prime}}{I H_{m}^{i} \varphi(z)}-\left(\frac{\left(I H_{m}^{i} \varphi(z)\right)^{\prime}}{I H_{m}^{i} \varphi(z)}\right)^{2}\right] \prec q(z),
$$

then

$$
z^{2} \frac{\left(I H_{m}^{i} \varphi(z)\right)^{\prime}}{\left(I H_{m}^{i} \varphi(z)\right)^{\prime}} \prec h(z)
$$

Proof. Putting

$$
p(z)=z^{2} \frac{\left(I H_{m}^{i} \varphi(z)\right)^{\prime}}{\left(I H_{m}^{i} \varphi(z)\right)^{\prime}}, p \in H[0,1] .
$$


A.R.S. Juma, R.A. Hameed, M.I. Hameed - Some Results of Second Order ...

By take the derivative of both sides, we get

$$
p(z)+\frac{z}{\tau} p(z)=z^{2} \frac{\tau+2}{\tau} \frac{\left(I H_{m}^{i} \varphi(z)\right)^{\prime}}{I H_{m}^{i} \varphi(z)}+\frac{z^{3}}{\tau}\left[\frac{\left(I H_{m}^{i} \varphi(z)\right)^{\prime}}{I H_{m}^{i} \varphi(z)}-\left(\frac{\left(I H_{m}^{i} \varphi(z)\right)^{\prime}}{I H_{m}^{i} \varphi(z)}\right)^{2}\right],
$$

thus, by aid of the relation (12) becomes

$$
p(z)+\frac{z}{\tau} p^{\prime}(z) \prec q(z)=h(z)+\frac{z}{\tau} h^{\prime}(z) .(z \in U)
$$

Therefore, with Lemma 2 we obtain

$$
p(z)=z^{2} \frac{\left(I H_{m}^{i} \varphi(z)\right)^{\prime}}{\left(I H_{m}^{i} \varphi(z)\right)} \prec h(z) .(z \in U)
$$

The result is sharp.

Theorem 2.7. If $q$ be a holomorphic function and it satisfies the inequality

$$
\operatorname{Re}\left\{1+\frac{z q^{\prime}(z)}{q(z)}\right\}>-1 / 2, \text { with } q(0)=1,
$$

where $\tau, i \geq 0, m \in N$.If $\varphi \in A$ and satisfies the following differential subordination

$$
z^{2} \frac{\tau+2}{\tau} \frac{\left(I H_{m}^{i} \varphi(z)\right)^{\prime}}{I H_{m}^{i} \varphi(z)}+\frac{z^{3}}{\tau}\left[\frac{\left(I H_{m}^{i} \varphi(z)\right)^{\prime}}{I H_{m}^{i} \varphi(z)}-\left(\frac{\left(I H_{m}^{i} \varphi(z)\right)^{\prime}}{I H_{m}^{i} \varphi(z)}\right)^{2}\right] \prec q(z),
$$

holds true, then

$$
z^{2} \frac{\left(I H_{m}^{i} \varphi(z)\right)^{\prime}}{\left(I H_{m}^{i} \varphi(z)\right)} \prec g(z)
$$

where $g(z)$ is given by

$$
g(z)=\frac{\tau}{z^{\tau}} \int_{0}^{z} q(t) t^{\tau-1} d t
$$

The function $g(z)$ is convex and it is the best dominant.

Proof. Putting

$$
p(z)=z^{2} \frac{\left(I H_{m}^{i} \varphi(z)\right)^{\prime}}{\left(I H_{m}^{i} \varphi(z)\right)^{\prime}}, p \in H[0,1] .(z \in U)
$$


A.R.S. Juma, R.A. Hameed, M.I. Hameed - Some Results of Second Order ...

By take the derivative of both sides, we have

$$
p(z)+\frac{z}{\tau} p(z)=z^{2} \frac{\tau+2}{\tau} \frac{\left(I H_{m}^{i} \varphi(z)\right)^{\prime}}{I H_{m}^{i} \varphi(z)}+\frac{z^{3}}{\tau}\left[\frac{\left(I H_{m}^{i} \varphi(z)\right)^{\prime}}{I H_{m}^{i} \varphi(z)}-\left(\frac{\left(I H_{m}^{i} \varphi(z)\right)^{\prime}}{I H_{m}^{i} \varphi(z)}\right)^{2}\right],
$$

thus, by aid of the relation (12) becomes

$$
p(z)+\frac{z}{\tau} p^{\prime}(z) \prec q(z)=h(z)+\frac{z}{\tau} h^{\prime}(z) .(z \in U)
$$

Therefore, with Lemma 1.1 we obtain

$$
p(z)=z^{2} \frac{\left(I H_{m}^{i} \varphi(z)\right)^{\prime}}{\left(I H_{m}^{i} \varphi(z)\right)} \prec g(z), \quad(z \in U)
$$

where

$$
g(z)=\frac{\tau}{z^{\tau}} \int_{0}^{z} q(t) t^{\tau-1} d t
$$

The function $g(z)$ is convex and it is the best dominant.

Theorem 2.8. If $h$ is a convex function with $h(0)=1$ such that $q$ the following function

$$
q(z)=h(z)+z h^{\prime}(z), \quad(z \in U)
$$

where $\tau, i \geq 0, m \in N$.If $\varphi \in A$ and satisfies the following differential subordination

$$
1-\frac{I H_{m}^{i} \varphi(z)\left(I H_{m}^{i} \varphi(z)\right)^{\prime}}{\left[\left(I H_{m}^{i} \varphi(z)\right)^{\prime}\right.} \prec q(z),
$$

then

$$
\frac{\left(I H_{m}^{i} \varphi(z)\right)}{z\left(I H_{m}^{i} \varphi(z)\right)^{\prime}} \prec h(z) .
$$

The result is sharp.

Proof. Putting

$$
p(z)=\frac{\left(I H_{m}^{i} \varphi(z)\right)}{z\left(I H_{m}^{i} \varphi(z)\right)^{\prime}}, p \in H[1,1] .
$$


A.R.S. Juma, R.A. Hameed, M.I. Hameed - Some Results of Second Order ...

By take the derivative of both sides, we have

$$
p(z)+z p^{\prime}(z)=1-\frac{I H_{m}^{i} \varphi(z)\left(I H_{m}^{i} \varphi(z)\right)^{\prime}}{\left[\left(I H_{m}^{i} \varphi(z)\right)^{\prime}\right]^{2}},
$$

thus, by aid of the relation (13) becomes

$$
p(z)+z p^{\prime}(z) \prec q(z)=h(z)+z h^{\prime}(z) .(z \in U)
$$

Therefore, with Lemma 1.2 we obtain

$$
p(z)=\frac{\left(I H_{m}^{i} \varphi(z)\right)}{z\left(I H_{m}^{i} \varphi(z)\right)^{\prime}} \prec h(z) .
$$

Proof. If $q$ be a holomorphic function and it satisfies the inequality

$$
\operatorname{Re}\left\{1+\frac{z q^{\prime}(z)}{q^{\prime}(z)}\right\}>-1 / 2, \text { with } q(0)=1,
$$

where $\tau, i \geq 0, m \in N$.If $\varphi \in A$ and satisfies the following differential subordination

$$
1-\frac{I H_{m}^{i} \varphi(z)\left(I H_{m}^{i} \varphi(z)\right)^{\prime}}{\left[\left(I H_{m}^{i} \varphi(z)\right)^{\prime}\right]^{2}} \prec q(z), \quad(z \in U)
$$

holds true, then

$$
\frac{\left(I H_{m}^{i} \varphi(z)\right)}{z\left(I H_{m}^{i} \varphi(z)\right)^{\prime}} \prec g(z) .
$$

where $g(z)$ is given by

$$
g(z)=\frac{1}{z} \int_{0}^{z} q(t) d t
$$

The function $g(z)$ is convex and it is the best dominant.

Putting

$$
p(z)=\frac{\left(I H_{m}^{i} \varphi(z)\right)}{z\left(I H_{m}^{i} \varphi(z)\right)^{\prime}}, p \in H[0,1] .(z \in U)
$$

By take the derivative of both sides, we get

$$
p(z)+z p^{\prime}(z)=1-\frac{I H_{m}^{i} \varphi(z)\left(I H_{m}^{i} \varphi(z)\right)^{\prime}}{\left[\left(I H_{m}^{i} \varphi(z)\right)^{\prime}\right]^{2}},
$$


A.R.S. Juma, R.A. Hameed, M.I. Hameed - Some Results of Second Order ...

thus, by aid of the relation (12) becomes

$$
p(z)+z p^{\prime}(z) \prec q(z)=h(z)+z h^{\prime}(z) .(z \in U)
$$

Therefore, with Lemma 1 we obtain

$$
p(z)=\frac{\left(I H_{m}^{i} \varphi(z)\right)}{z\left(I H_{m}^{i} \varphi(z)\right)^{\prime}} \prec g(z), \quad(z \in U)
$$

where

$$
g(z)=\frac{1}{z} \int_{0}^{z} q(t) d t
$$

The function $g(z)$ is convex and it is the best dominant.

Theorem 2.9. If the function $q$ defined by

$$
q(z)=\frac{1+(2 \alpha-1) z}{1+z},(0 \leq \alpha<1)
$$

is a convex function in $U$, where $i \geq 0, m \in N$.If $\varphi \in A$ and the following differential subordination

$$
1-\frac{I H_{m, \beta}^{i} \varphi(z)\left(I H_{m, \beta}^{i} \varphi(z)\right)^{\prime}}{\left[\left(I H_{m, \beta}^{i} \varphi(z)\right)^{2}\right.} \prec q(z)
$$

holds true, then

$$
\frac{\left(I H_{m, \beta}^{i} \varphi(z)\right)}{z\left(I H_{m, \beta}^{i} \varphi(z)\right)^{\prime}} \prec g(z),
$$

where

$$
g(z)=(2 \alpha-1)+2(1-\alpha) \frac{\ln (1+z)}{z} .
$$

The function $g(z)$ is convex and it is the best dominant.

Proof. Putting

$$
p(z)=\frac{\left(I H_{m, \beta}^{i} \varphi(z)\right)}{z\left(I H_{m, \beta}^{i} \varphi(z)\right)^{\prime}}
$$


A.R.S. Juma, R.A. Hameed, M.I. Hameed - Some Results of Second Order ...

thus, by aid of the relation (14) becomes

$$
p(z)+z p^{\prime}(z) \prec q(z)=\frac{1+(2 \alpha-1) z}{1+z},(0 \leq \alpha<1)
$$

therefore, with Lemma 1.1 for $\gamma=1$, we get

$$
\begin{aligned}
& \frac{\left(I H_{m, \beta}^{i} \varphi(z)\right)}{z\left(I H_{m, \beta}^{i} \varphi(z)\right)^{\prime}} \prec g(z)=\frac{1}{z} \int_{0}^{z} q(t) d t=\frac{1}{z} \int_{0}^{z} \frac{1+(2 \alpha-1) t}{1+t} d t \\
= & \frac{1}{z} \int_{0}^{z}\left[(2 \alpha-1)+\frac{2(1-\alpha)}{1+t}\right] d t=(2 \alpha-1)+2(1-\alpha) \frac{\ln (1+z}{z} .
\end{aligned}
$$

Theorem 2.10. If $h$ is a convex function with $h(0)=1$ such that $q$ the following function

$$
q(z)=h(z)+z h^{\prime}(z), \quad(z \in U)
$$

where $i \geq 0, m \in N$.If $\varphi \in A$ and satisfies the following differential subordination

$$
\left[\left(I H_{m}^{i} \varphi(z)\right)^{\prime}\right]^{2}+I H_{m}^{i} \varphi(z)\left(I H_{m}^{i} \varphi(z)\right)^{\prime} \prec q(z),
$$

then

$$
\frac{\left(I H_{m}^{i} \varphi(z)\right)\left(I H_{m}^{i} \varphi(z)\right)^{\prime}}{z} \prec h(z)
$$

Proof. Putting

$$
p(z)=\frac{\left(I H_{m}^{i} \varphi(z)\right)\left(I H_{m}^{i} \varphi(z)\right)^{\prime}}{z}, p \in H[0,1] .
$$

By take the derivative of both sides, we have

$$
p(z)+z p^{\prime}(z)=\left[\left(I H_{m}^{i} \varphi(z)\right)^{\prime}\right]^{2}+I H_{m}^{i} \varphi(z)\left(I H_{m}^{i} \varphi(z)\right)^{\prime},
$$

thus, by aid of the relation (15) becomes

$$
p(z)+z p^{\prime}(z) \prec q(z)=h(z)+z h^{\prime}(z) .(z \in U)
$$

Therefore, with Lemma 2 we get

$$
p(z)=\frac{\left(I H_{m}^{i} \varphi(z)\right)\left(I H_{m}^{i} \varphi(z)\right)^{\prime}}{z} \prec h(z) .
$$

The result is sharp. 
A.R.S. Juma, R.A. Hameed, M.I. Hameed - Some Results of Second Order ...

Theorem 2.11. If $q$ be a holomorphic function and it satisfies the inequality

$$
\operatorname{Re}\left\{1+\frac{z q^{\prime}(z)}{q^{\prime}(z)}\right\}>-1 / 2, \text { with } q(0)=1,
$$

where $i \geq 0, m \in N$.If $\varphi \in A$ and satisfies the following differential subordination

$$
\left[\left(I H_{m}^{i} \varphi(z)\right)^{2}\right]^{2}+I H_{m}^{i} \varphi(z)\left(I H_{m}^{i} \varphi(z)\right)^{\prime} \prec q(z), \quad(z \in U)
$$

holds true, then

$$
\frac{\left(I H_{m}^{i} \varphi(z)\right)\left(I H_{m}^{i} \varphi(z)\right)^{\prime}}{z} \prec g(z)
$$

where $g(z)$ is given by

$$
g(z)=\frac{1}{z} \int_{0}^{z} q(t) d t
$$

The function $g(z)$ is convex and it is the best dominant.

Proof. Putting

$$
p(z)=\frac{\left(I H_{m}^{i} \varphi(z)\right)\left(I H_{m}^{i} \varphi(z)\right)^{\prime}}{z}, p \in H[0,1] .(z \in U)
$$

By take the derivative of both sides, we have

$$
p(z)+z p^{\prime}(z)=\left[\left(I H_{m}^{i} \varphi(z)\right)^{2}+I H_{m}^{i} \varphi(z)\left(I H_{m}^{i} \varphi(z)\right)^{\prime},\right.
$$

thus, by aid of the relation (15) becomes

$$
p(z)+z p^{\prime}(z) \prec q(z)=h(z)+z h^{\prime}(z) .(z \in U)
$$

Therefore, with Lemma 1.1 we obtain

$$
p(z)=\frac{\left(I H_{m}^{i} \varphi(z)\right)\left(I H_{m}^{i} \varphi(z)\right)^{\prime}}{z} \prec g(z), \quad(z \in U)
$$

where

$$
g(z)=\frac{1}{z} \int_{0}^{z} q(t) d t
$$

The function $g(z)$ is convex and it is the best dominant. 
A.R.S. Juma, R.A. Hameed, M.I. Hameed - Some Results of Second Order ...

Theorem 2.12. If the function $q$ defined by

$$
q(z)=\frac{1+(2 \alpha-1) z}{1+z},(0 \leq \alpha<1)
$$

is a convex function in $U$, where $i \geq 0, m \in N$.If $\varphi \in A$ and the following differential subordination

$$
\left[\left(I H_{m}^{i} \varphi(z)\right)^{\prime}\right]^{2}+I H_{m}^{i} \varphi(z)\left(I H_{m}^{i} \varphi(z)\right)^{\prime}, \prec q(z)
$$

holds true, then

$$
\frac{\left(I H_{m}^{i} \varphi(z)\right)\left(I H_{m}^{i} \varphi(z)\right)^{\prime}}{z} \prec g(z)
$$

where

$$
g(z)=(2 \alpha-1)+2(1-\alpha) \frac{\ln (1+z)}{z} .
$$

The function $g(z)$ is convex and it is the best dominant.

Proof. Let

$$
p(z)=\frac{\left(I H_{m}^{i} \varphi(z)\right)\left(I H_{m}^{i} \varphi(z)\right)^{\prime}}{z}
$$

thus, by aid of the relation (15) becomes

$$
p(z)+z p^{\prime}(z) \prec q(z)=\frac{1+(2 \alpha-1) z}{1+z},(0 \leq \alpha<1)
$$

therefore, with Lemma 1.1 for $\gamma=1$, we obtain

$$
\begin{array}{r}
p(z)=\frac{\left(I H_{m}^{i} \varphi(z)\right)\left(I H_{m}^{i} \varphi(z)\right)^{\prime}}{z} \prec g(z)=\frac{1}{z} \int_{0}^{z} q(t) d t=\frac{1}{z} \int_{0}^{z} \frac{1+(2 \alpha-1) t}{1+t} d t \\
=\frac{1}{z} \int_{0}^{z}\left[(2 \alpha-1)+\frac{2(1-\alpha)}{1+t}\right] d t=(2 \alpha-1)+2(1-\alpha) \frac{\ln (1+z}{z} .
\end{array}
$$

Theorem 2.13. If $h$ is a convex function with $h(0)=1$ such that $q$ the following function

$$
q(z)=h(z)+\frac{z}{1-\tau} h^{\prime}(z), \quad(z \in U)
$$


A.R.S. Juma, R.A. Hameed, M.I. Hameed - Some Results of Second Order ...

where $\tau \in(0,1), i \geq 0, m \in N$.If $\varphi \in A$ and satisfies the following differential subordination

$$
\left(\frac{z}{I H_{m}^{i} \varphi(z)}\right)^{\tau} \frac{I H_{m}^{i}\left[\alpha_{1}+1\right] \varphi(z)}{1-\tau}\left[\frac{\left(I H_{m}^{i}\left[\alpha_{1}+1\right] \varphi(z)\right)^{\prime}}{I H_{m}^{i}\left[\alpha_{1}+1\right] \varphi(z)}-\tau \frac{\left(I H_{m}^{i} \varphi(z)\right)^{\prime}}{I H_{m}^{i} \varphi(z)}\right] \prec q(z),
$$

then

$$
\frac{\left(I H_{m}^{i}\left[\alpha_{1}+1\right] \varphi(z)\right.}{z}\left(\frac{z}{I H_{m}^{i} \varphi(z)}\right)^{\tau} \prec h(z) .
$$

Proof. Putting

$$
p(z)=\frac{\left(I H_{m}^{i}\left[\alpha_{1}+1\right] \varphi(z)\right.}{z}\left(\frac{z}{I H_{m}^{i} \varphi(z)}\right)^{\tau}, p \in H[1,1] .
$$

By take the derivative of both sides, we have

$$
p(z)+\frac{z}{1-\tau} p^{\prime}(z)=\left(\frac{z}{I H_{m}^{i} \varphi(z)}\right)^{\tau} \frac{I H_{m}^{i}\left[\alpha_{1}+1\right] \varphi(z)}{1-\tau}\left[\frac{\left(I H_{m}^{i}\left[\alpha_{1}+1\right] \varphi(z)\right)^{\prime}}{I H_{m}^{i}\left[\alpha_{1}+1\right] \varphi(z)}-\tau \frac{\left(I H_{m}^{i} \varphi(z)\right)^{\prime}}{I H_{m}^{i} \varphi(z)}\right]
$$

thus, by aid of the relation (16) becomes

$$
p(z)+\frac{z}{1-\tau} p^{\prime}(z) \prec q(z)=h(z)+\frac{z}{1-\tau} h^{\prime}(z) .(z \in U)
$$

Therefore, with Lemma 1.2 we obtain

$$
p(z)=\frac{\left(I H_{m}^{i}\left[\alpha_{1}+1\right] \varphi(z)\right.}{z}\left(\frac{z}{I H_{m}^{i} \varphi(z)}\right)^{\tau} \prec h(z) .
$$

The result is sharp.

Theorem 2.14. If $q$ be a holomorphic function and it satisfies the inequality

$$
\operatorname{Re}\left\{1+\frac{z q^{\prime \prime}(z)}{q(z)}\right\}>-1 / 2, \text { with } q(0)=1,
$$

where $\tau \in(0,1), i \geq 0, m \in N$.If $\varphi \in A$ and satisfies the following differential subordination

$$
\left(\frac{z}{I H_{m}^{i} \varphi(z)}\right)^{\tau} \frac{I H_{m}^{i}\left[\alpha_{1}+1\right] \varphi(z)}{1-\tau}\left[\frac{\left(I H_{m}^{i}\left[\alpha_{1}+1\right] \varphi(z)\right)^{\prime}}{I H_{m}^{i}\left[\alpha_{1}+1\right] \varphi(z)}-\tau \frac{\left(I H_{m}^{i} \varphi(z)\right)^{\prime}}{I H_{m}^{i} \varphi(z)}\right] \prec q(z), \quad(z \in U)
$$

holds true, then

$$
\frac{\left(I H_{m}^{i}\left[\alpha_{1}+1\right] \varphi(z)\right.}{z}\left(\frac{z}{I H_{m}^{i} \varphi(z)}\right)^{\tau} \prec g(z),
$$


A.R.S. Juma, R.A. Hameed, M.I. Hameed - Some Results of Second Order ...

where $g(z)$ is given by

$$
g(z)=\frac{1-\tau}{z^{1-\tau}} \int_{0}^{z} q(t) t^{-\tau} d t
$$

The function $g(z)$ is convex and it is the best dominant.

Proof. Putting

$$
p(z)=\frac{\left(I H_{m}^{i}\left[\alpha_{1}+1\right] \varphi(z)\right.}{z}\left(\frac{z}{I H_{m}^{i} \varphi(z)}\right)^{\tau}, p \in H[0,1] .(z \in U)
$$

By take the derivative of both sides, we have

$p(z)+\frac{z}{1-\tau} p^{\prime}(z)=\left(\frac{z}{I H_{m}^{i} \varphi(z)}\right)^{\tau} \frac{I H_{m}^{i}\left[\alpha_{1}+1\right] \varphi(z)}{1-\tau}\left[\frac{\left(I H_{m}^{i}\left[\alpha_{1}+1\right] \varphi(z)\right)^{\prime}}{I H_{m}^{i}\left[\alpha_{1}+1\right] \varphi(z)}-\tau \frac{\left(I H_{m}^{i} \varphi(z)\right)^{\prime}}{I H_{m}^{i} \varphi(z)}\right]$,

thus, by aid of the relation (16) becomes

$$
p(z)+\frac{z}{1-\tau} p^{\prime}(z) \prec q(z)=h(z)+\frac{z}{1-\tau} h^{\prime}(z) .(z \in U)
$$

Therefore, with Lemma 1 we obtain

$$
p(z)=\frac{\left(I H_{m}^{i}\left[\alpha_{1}+1\right] \varphi(z)\right.}{z}\left(\frac{z}{I H_{m}^{i} \varphi(z)}\right)^{\tau} \prec g(z), \quad(z \in U)
$$

where

$$
g(z)=\frac{1-\tau}{z^{1-\tau}} \int_{0}^{z} q(t) t^{-\tau} d t
$$

The function $g(z)$ is convex and it is the best dominant

\section{REFERENCES}

[1] M. H. Al-Abbadi and M. Darus, Differential subordination defined by new generalised derivative operator for analytic functions, Int. J. Math. Math. Sci., Vol. 2010, (2010), P. 15.

[2] W. Becken and P. Schmelcher, The analytic continuation of the Gaussian hypergeometric function $2 F 1(a, b ; c$; $z$ ) for arbitrary parameters, Journal of Computational and Applied Mathematics, 126, (2000), 449-478.

[3] T. Bulboaca, Differential subordinations and superordinations, Recent Results, Casa Cartii de Stiinta, Cluj-Napoca, (2005). 
A.R.S. Juma, R.A. Hameed, M.I. Hameed - Some Results of Second Order ...

[4] M. Darus and K. Al-Shaqsi, Differential sandwich theorems with generalised derivative operator, Int. J. Comput. Math. Sci., Vol. 2, No. 2, (2008), 75-78.

[5] J. Dziok and H.M. Srivastava, Classes of analytic functions associated with the generalized hypergeometric function, Appl. Math. Comput., 103, (1999), 1-3.

[6] D. J. Hallenbeck and S. Ruscheweyh, Subordination by convex functions, Proc. Amer. Math. Soc., 52, (1975), 191-195.

[7] A. R. S. Juma, M. S. A. Hussein and M. F. Hani, On second-order differential subordination and superordination of analytic and multivalent functions, International Journal of Recent Scientific Research, Vol 6, May 2015, 5 pages.

[8] A.R.S.Juma, S.R.Kulkarni, On univalent functions with negative coefficients by using generalized Salagean operator, Filomat,21, (2007), 173-184.

[9] S. S. Miller, P. T. Mocanu, Differential subordinations, Theory and applications, Monographs and Textbooks in Pure and Applied Mathematics, Marcel Dekker, Inc., New York, (2000).

[10] P. T. Mocanu, T. Bulboaca and G. Salagean, Geometric theory of univalent functions, Casa Cartii de Stiinta, Cluj-Napoca, (1999).

[11] G. Murugusundaramoorthy, A.R.S.Juma and S.R.kulkarni, Convolution properties of univalent functions defined generalized Salagean operator, JMA, 30, (2008), 103-112.

[12] G. I. Oros, On a class of holomorphic functions defined by the Ruscheweyh derivative, Int. J. Math. Math. Sci., Vol. 2003, No. 65, (2003), 4139-4144.

[13] G. I. Oros, G. Oros, I. H. Kim and N. E. Cho, Differential subordination associated with the Dziok-Srivastava operator, Math. Reports, 1, (2011), 57-64.

[14] K. Al-Shaqsi and M. Darus, On univalent functions with respect to k-symmetric points defined by a generalized Ruscheweyh derivatives operator, J. Anal. Appl., Vol. 7, No. 1, (2009), 53-61.

[15] H.M. Srivastava and A. A. Attiya, An integral operator associated with the HurwitzLerch Zeta function and differential subordination, Integral Transforms and Special Functions, 18, (2007), 207-216.

Abdul Rahman S. Juma

College of Education for Pure Sciences

Department of Mathematics

University of Anbar -IRAQ

e-mail:dr_juma@hotmail.com

Raad Awad Hameed

College of Education for Pure Sciences 
A.R.S. Juma, R.A. Hameed, M.I. Hameed - Some Results of Second Order ...

Department of Mathematics

University of Tikrit -IRAQ

e-mail: awad.raad2@gmail.com

Mustafa Ibrahim Hameed

College of Education for Pure Sciences

Department of Mathematics

University of Tikrit -IRAQ

e-mail: mustafa8095@yahoo.com 